Bull. Korean Math. Soc. 50 (2013), No. 6, pp. 1973-1979

http://dx.doi.org/10.4134/BKMS.2013.50.6.1973

\title{
THE WINTNER THEOREM IN UNITAL COMPLETE RANDOM NORMED ALGEBRAS
}

\author{
YUEHAN TANG
}

\begin{abstract}
The main purpose of this paper is to give the Wintner theorem in unital complete random normed algebras which is a random generalization of the classical Wintner theorem in Banach algebras. As an application of the Wintner theorem in unital complete random normed algebras, we also obtain that the identity operator on a complete random normed module is not a commutator.
\end{abstract}

\section{Introduction}

The Wintner theorem is important in classical Banach algebras which has attracted many authors' attention since it is proved in 1947 by Wintner [19]. The famous theorem is closely related to the study of commutators of operators [18].

The notion of a random normed module (briefly, an $R N$ module) was presented in [2] and subsequently elaborated in [6], which is a random generalization of that of a normed space. The theory of random conjugate spaces for $R N$ modules is a powerful tool for the deep development of $R N$ modules. The theory of $R N$ modules together with their random conjugate spaces has undergone a systematic and deep development $[3,5,11,12]$ and found many important applications to various topics, e.g., in best approximation $[11,13]$, in representation of the dual of Lebesgue-Bochner function spaces [7], in geometry of Banach spaces [3], in the study of measurability problems [8] and in particular also in the recent study of conditional risk measures $[9,10]$. Banach algebra is an important part of functional analysis [14], thus we recently introduced the notion of random normed algebras in the framework of $R N$ modules and further established some basic facts on complete random normed algebras $[15,16,17]$.

Based on the work of [17], in this paper we will establish the Wintner theorem in a unital complete random normed algebra. Precisely speaking, we first give

Received July 27, 2012.

2010 Mathematics Subject Classification. 46H25, 46H05, 15A78.

Key words and phrases. random normed module, random normed algebra, random spectrum, Wintner theorem.

Supported by the National Natural Science Foundation of China (No. 11301225). 
the relationship between the random spectra of $x y$ and $y x$ and then, as a random generalization of the classical Wintner theorem, we obtain the Wintner theorem in a unital complete random normed algebra by use of the relationship and the random spectral radius formula established in [17]. As an application of the Wintner theorem in unital complete random normed algebras, we also obtain that the identity operator on a complete random normed module is not a commutator.

The remainder of this paper is organized as follows: in Section 2 we give some necessary terminology and notation and in Section 3 we give the main results and their proofs.

\section{Preliminaries}

Throughout this paper, $N$ denotes the set of positive integers, $K$ the scalar field $R$ of real numbers or $C$ of complex numbers, $(\Omega, \mathcal{F}, P)$ a probability space, $\bar{L}^{0}(\mathcal{F}, R)$ the set of equivalence classes of extended real-valued $\mathcal{F}$-random variables on $\Omega, L^{0}(\mathcal{F}, K)$ the algebra of equivalence classes of $K$-valued $\mathcal{F}$-random variables on $\Omega$.

It is well known from [1] that $\bar{L}^{0}(\mathcal{F}, R)$ is a complete lattice under the ordering $\leqslant: \quad \xi \leqslant \eta$ if and only if $\xi^{0}(\omega) \leqslant \eta^{0}(\omega)$ for $P$-almost all $\omega$ in $\Omega$ (briefly, a.s.), where $\xi^{0}$ and $\eta^{0}$ are arbitrarily chosen representatives of $\xi$ and $\eta$, respectively. Furthermore, every subset $A$ of $\bar{L}^{0}(\mathcal{F}, R)$ has a supremum, denoted by $\vee A$, and an infimum, denoted by $\wedge A$, and there exist two sequences $\left\{a_{n}, n \in N\right\}$ and $\left\{b_{n}, n \in N\right\}$ in $A$ such that $\vee_{n \geqslant 1} a_{n}=\vee A$ and $\wedge_{n \geqslant 1} b_{n}=$ $\wedge A$. If, in addition, $A$ is directed (accordingly, dually directed), then the above $\left\{a_{n}, n \in N\right\}$ (accordingly, $\left\{b_{n}, n \in N\right\}$ ) can be chosen as nondecreasing (accordingly, nonincreasing). Finally $L^{0}(\mathcal{F}, R)$, as a sublattice of $\bar{L}^{0}(\mathcal{F}, R)$, is complete in the sense that every subset with an upper bound has a supremum (equivalently, every subset with a lower bound has an infimum).

Specially, let $\bar{L}_{+}^{0}=\left\{\xi \in \bar{L}^{0}(\mathcal{F}, R) \mid \xi \geqslant 0\right\}$ and $L_{+}^{0}=\left\{\xi \in L^{0}(\mathcal{F}, R) \mid \xi \geqslant 0\right\}$.

Besides the equivalence classes of $\mathcal{F}$-random variables, we also use the equivalence classes of $\mathcal{F}$-measurable sets. Let $A \in \mathcal{F}$, then the equivalence class of $A$, denoted by $\tilde{A}$, is defined by $\tilde{A}=\{B \in \mathcal{F}: P(A \triangle B)=0\}$, where $A \triangle B=(A \backslash B) \cup(B \backslash A)$ is the symmetric difference of $A$ and $B$, and $P(\tilde{A})$ is defined to be $P(A)$.

As usual, we also make the following convention: for any $\xi \in L^{0}(\mathcal{F}, C)$, $[\xi=0]$ and $[\xi \neq 0]$ stand for the equivalence classes of the $\mathcal{F}$-measurable set $\left\{\omega \in \Omega: \xi^{0}(\omega)=0\right\}$ and $\left\{\omega \in \Omega: \xi^{0}(\omega) \neq 0\right\}$, respectively, where $\xi^{0}$ is an arbitrarily selected representative of $\xi$.

Definition 2.1 $([6])$. An ordered pair $(S,\|\cdot\|)$ is called a random normed module (briefly, an $R N$ module) over $K$ with base $(\Omega, \mathcal{F}, P)$ if $S$ is a left module over the algebra $L^{0}(\mathcal{F}, K)$ and $\|\cdot\|$ is a mapping from $\mathrm{S}$ to $L_{+}^{0}$ such that the following conditions are satisfied:

$(R N M-1)\|\xi x\|=|\xi|\|x\|, \forall \xi \in L^{0}(\mathcal{F}, K), x \in S ;$ 
$(R N M-2)\|x+y\| \leq\|x\|+\|y\|, \forall x, y \in S$

$(R N M-3)\|x\|=0$ implies $x=0$ (the zero element in $S$ ).

Where $\|x\|$ is called the $L^{0}$-norm of the vector $x$ in $S$.

In this paper, given an $R N$ module $(S,\|\cdot\|)$ over $K$ with base $(\Omega, \mathcal{F}, P)$, it is always assumed that $(S,\|\cdot\|)$ is endowed with its $(\epsilon, \lambda)$-topology: for any $\epsilon>0,0<\lambda<1$, let $N(\epsilon, \lambda)=\{x \in S \mid P\{\omega \in \Omega:\|x\|(\omega)<\epsilon\}>1-\lambda\}$, then the family $\mathcal{U}_{0}=\{N(\epsilon, \lambda) \mid \epsilon>0,0<\lambda<1\}$ forms a local base at the null element 0 of some metrizable linear topology for $S$, called the $(\epsilon, \lambda)$-topology for $S$.

Definition $2.2([17])$. An ordered pair $(S,\|\cdot\|)$ is called a random normed algebra (briefly, an $R N$ algebra) over $K$ with base $(\Omega, \mathcal{F}, P)$ if $(S,\|\cdot\|)$ is an $R N$ module over $K$ with base $(\Omega, \mathcal{F}, P)$ and also a ring such that the following two conditions are satisfied:

(1) $(\xi \cdot x) y=x(\xi \cdot y)=\xi \cdot(x y)$ for all $\xi \in L^{0}(\mathcal{F}, K)$ and all $x, y \in S$;

(2) the $L^{0}$-norm $\|\cdot\|$ is submultiplicative, that is, $\|x y\| \leq\|x\|\|y\|$ for all $x, y \in S$.

Furthermore, the $R N$ algebra is said to be unital if it has the identity element $e$ and $\|e\|=1$. As usual, the $R N$ algebra $(S,\|\cdot\|)$ is said to be complete if the $R N$ module $(S,\|\cdot\|)$ is complete.

Example 2.1 ([17]). Let $(X,\|\cdot\|)$ be a normed algebra over $C$ and $L^{0}(\mathcal{F}, X)$ be the $R N$ module of equivalence classes of $X$-valued $\mathcal{F}$-random variables on $(\Omega, \mathcal{F}, P)$. Define a multiplication $\cdot: L^{0}(\mathcal{F}, X) \times L^{0}(\mathcal{F}, X) \rightarrow L^{0}(\mathcal{F}, X)$ by $x \cdot y=$ the equivalence class determined by the $\mathcal{F}$-random variable $x^{0} y^{0}$, which is defined by $\left(x^{0} y^{0}\right)(\omega)=\left(x^{0}(\omega)\right) \cdot\left(y^{0}(\omega)\right), \forall \omega \in \Omega$, where $x^{0}$ and $y^{0}$ are arbitrarily chosen representatives of $x$ and $y$ in $L^{0}(\mathcal{F}, X)$, respectively. Then $\left(L^{0}(\mathcal{F}, X),\|\cdot\|\right)$ is an $R N$ algebra, in particular $L^{0}(\mathcal{F}, C)$ is a unital $R N$ algebra with identity 1 .

Definition $2.3([17])$. Let $(S,\|\cdot\|)$ be an $R N$ algebra with identity $e$ over $C$ with base $(\Omega, \mathcal{F}, P)$, and $A$ be any given element in $\mathcal{F}$ such that $P(A)>0$. An element $x \in S$ is invertible on $A$ if there exists $y \in S$ such that $\tilde{I}_{A} \cdot x y=$ $\tilde{I}_{A} \cdot y x=\tilde{I}_{A} \cdot e$. Clearly, $\tilde{I}_{A} \cdot y$ is unique and called the inverse on $A$ of $x$, denoted by $x_{A}^{-1}$. Let $G(S, A)$ denote the set of elements of $S$ which are invertible on $A$. Then $\tilde{I}_{A} \cdot G(S, A)$ is also a group, and $(x y)_{A}^{-1}=y_{A}^{-1} x_{A}^{-1}$ for any $x$ and $y$ in $\tilde{I}_{A} \cdot G(S, A)$. For any $x \in S$, the sets

$$
\begin{aligned}
\sigma(x, S, A) & =\left\{\xi \in L^{0}(\mathcal{F}, C): \tilde{I}_{A} \cdot(\xi \cdot e-x) \notin \tilde{I}_{A} \cdot G(S, A)\right\}, \\
\sigma(x, S) & =\bigcap_{A \in \mathcal{F}, P(A)>0} \sigma(x, S, A)
\end{aligned}
$$

are called the random spectrum on $A$ of $x$ in $S$ and the random spectrum of $x$ in $S$, respectively, and further their complements $\rho(x, S, A)=L^{0}(\mathcal{F}, C) \backslash \sigma(x, S, A)$ and $\rho(x, S)=L^{0}(\mathcal{F}, C) \backslash \sigma(x, S)$ are called the random resolvent set on $A$ of $x$ and the random resolvent set of $x$, respectively. 
Definition $2.4([17])$. Let $(S,\|\cdot\|)$ be an $R N$ algebra with identity $e$ over $C$ with base $(\Omega, \mathcal{F}, P)$. For any $x \in S, r(x)=\vee\{|\xi|: \xi \in \sigma(x, S)\}$ is called the random spectral radius of $x$.

Besides, $\wedge\left\{\left\|x^{n}\right\|^{\frac{1}{n}} \mid n \in N\right\}$ is denoted by $r_{p}(x)$, for any $x$ in an $R N$ algebra over $K$ with base $(\Omega, \mathcal{F}, P)$.

\section{Main results and proofs}

Lemma 3.1. Let $(S,\|\cdot\|)$ be an $R N$ algebra with identity e over $C$ with base $(\Omega, \mathcal{F}, P)$. For any $x$ and $y$ in $S$, we have the following:

$$
\begin{aligned}
& \sigma(x y, S) \backslash\left\{\xi \in L^{0}(\mathcal{F}, C) \mid P([\xi=0])>0\right\} \\
= & \sigma(y x, S) \backslash\left\{\xi \in L^{0}(\mathcal{F}, C) \mid P([\xi=0])>0\right\} .
\end{aligned}
$$

Proof. For any $x$ and $y$ in $S$, if $\xi \notin \sigma(x y, S)$ and $\xi \notin\left\{\xi \in L^{0}(\mathcal{F}, C) \mid P([\xi=\right.$ $0])>0\}$, then there exist $A \in \mathcal{F}$ and $z \in S$ such that $P(A)>0$ and $\left[\tilde{I}_{A} \cdot(\xi\right.$. $e-x y)] z=z\left[\tilde{I}_{A} \cdot(\xi \cdot e-x y)\right]=\tilde{I}_{A} \cdot e$.

It follows that $\tilde{I}_{A} \cdot(x y z)=\left(\tilde{I}_{A} \xi\right) \cdot z-\tilde{I}_{A} \cdot e=\tilde{I}_{A} \cdot(z x y)$, and hence

$$
\begin{aligned}
& {\left[\tilde{I}_{A} \cdot(\xi \cdot e-y x)\right]\left[\tilde{I}_{A} \cdot(e+y z x)\right] } \\
= & \left(\tilde{I}_{A} \xi\right) \cdot e-\tilde{I}_{A} \cdot(y x)+\left(\tilde{I}_{A} \xi\right) \cdot(y z x)-y\left[\tilde{I}_{A} \cdot(x y z)\right] x \\
= & \left(\tilde{I}_{A} \xi\right) \cdot e-\tilde{I}_{A} \cdot(y x)+\left(\tilde{I}_{A} \xi\right) \cdot(y z x)-y\left[\tilde{I}_{A} \cdot(z x y)\right] x \\
= & \left(\tilde{I}_{A} \xi\right) \cdot e-\tilde{I}_{A} \cdot(y x)+y z\left[\tilde{I}_{A} \cdot(\xi \cdot e-x y)\right] x \\
= & \left(\tilde{I}_{A} \xi\right) \cdot e-\tilde{I}_{A} \cdot(y x)+y\left(\tilde{I}_{A} \cdot e\right) x \\
= & \left(\tilde{I}_{A} \xi\right) \cdot e .
\end{aligned}
$$

Similarly, $\left[\tilde{I}_{A} \cdot(e+y z x)\right]\left[\tilde{I}_{A} \cdot(\xi \cdot e-y x)\right]=\left(\tilde{I}_{A} \xi\right) \cdot e$.

It follows that $\left(\tilde{I}_{A} \xi^{-1}\right) \cdot(e+y z x)$ is the inverse on $A$ of $\tilde{I}_{A} \cdot(\xi \cdot e-y x)$, and hence $\xi \notin \sigma(y x, S)$. So $\left.\sigma(x y, S) \backslash\left\{\xi \in L^{0}(\mathcal{F}, C)\right) \mid P([\xi=0])>0\right\} \supseteq$ $\left.\sigma(y x, S) \backslash\left\{\xi \in L^{0}(\mathcal{F}, C)\right) \mid P([\xi=0])>0\right\}$. Since $x$ and $y$ are arbitrary, the reverse inclusion is an immediate consequence.

The proof of Lemma 3.2 below is very similar to Lemma 3.1, and thus it is omitted.

Lemma 3.2. Let $(S,\|\cdot\|)$ be an $R N$ algebra with identity e over $C$ with base $(\Omega, \mathcal{F}, P)$. For any $\xi \in L^{0}(\mathcal{F}, C)$, let $\tilde{A}=[\xi \neq 0]$, where $A$ is a representative element of $[\xi \neq 0]$. If $P(A)>0$, then for any $x$ and $y$ in $S, \xi \in \sigma(x y, S, A)$ if and only if $\xi \in \sigma(y x, S, A)$.

The following Lemma 3.3 is about the random spectral radius formula established in [17].

Lemma $3.3([17])$. Let $(S,\|\cdot\|)$ be a unital complete $R N$ algebra with identity e over $C$ with base $(\Omega, \mathcal{F}, P)$. Then for any $x \in S, \sigma(x, S)$ is a nonempty closed set and $r(x)=r_{p}(x)$. 
Using Lemma 3.1, Lemma 3.2 and Lemma 3.3, we obtain the Wintner theorem in unital complete random normed algebras as follows.

Theorem 3.1. Let $(S,\|\cdot\|)$ be a unital complete $R N$ algebra with identity $e$ over $C$ with base $(\Omega, \mathcal{F}, P)$. Then there exist no $x$ and $y$ in $S$ such that $x y-y x=e$.

Proof. Otherwise, if there exist $x$ and $y$ in $S$ such that $x y-y x=e$, and hence $x y=e+y x$, then we first prove that

$$
\left\{\xi \in L^{0}(\mathcal{F}, C) \mid P([\xi=0])>0\right\} \nsubseteq \sigma(x y, S) \bigcup \sigma(y x, S) .
$$

By the definition of the random spectrum, we obtain

$$
\sigma(x y, S)=\sigma(y x, S)+1(=\{\xi+1 \mid \xi \in \sigma(y x, S)\}) .
$$

For any $\xi \in \sigma(y x, S)$, let $\tilde{A}=[\xi=0]$, where $A$ is a representative element of $[\xi=0]$. If $P(A)>0$, then for any $B \in \mathcal{F}$ such that $B \subseteq A$ and $P(B)>0$, we have $\xi+1 \in \sigma(x y, S, B)$, thus by Lemma 3.2 we have $\xi+1 \in \sigma(y x, S, B)$, and hence $\xi+n \in \sigma(y x, S, B)(n=1,2, \ldots)$. It follows that

$$
n \tilde{I}_{A} \in \sigma\left(\tilde{I}_{A} \cdot(y x), \tilde{I}_{A} \cdot S\right)(n=1,2, \ldots) .
$$

By Lemma 3.3, we have

$$
n \tilde{I}_{A} \leq \tilde{I}_{A}\|y x\| \in L_{+}^{0}(\mathcal{F})(n=1,2, \ldots),
$$

which is a contradiction.

Similarly, for any $\eta \in \sigma(x y, S)$, let $\tilde{D}=[\eta=0]$, where $D$ is a representative element of $[\eta=0]$, if $P(D)>0$, then for any $E \in \mathcal{F}$ such that $E \subseteq D$ and $P(E)>0$, we have $\eta-1 \in \sigma(x y, S, E)$, further, by Lemma 3.2 we have $\eta-1 \in \sigma(y x, S, E)$, and hence

$$
\eta-n \in \sigma(x y, S, E)(n=1,2, \ldots) \text {. }
$$

It follows that

$$
-n \tilde{I}_{D} \in \sigma\left(\tilde{I}_{D} \cdot(x y), \tilde{I}_{D} \cdot S\right)(n=1,2, \ldots) .
$$

By Lemma 3.3, we have

$$
n \tilde{I}_{D} \leq \tilde{I}_{D}\|x y\| \in L_{+}^{0}(n=1,2, \ldots)
$$

which is also a contradiction. Therefore we have

$$
\left\{\xi \in L^{0}(\mathcal{F}, C) \mid P([\xi=0])>0\right\} \nsubseteq \sigma(x y, S) \bigcup \sigma(y x, S) .
$$

For any $\xi \in \sigma(y x, S)$, from $x y=e+y x$ we have $\xi+1 \in \sigma(x y, S)$. Again by Lemma 3.1, we have $\xi+1 \in \sigma(y x, S)$, and hence

$$
\xi+n \in \sigma(y x, S)(n=1,2, \ldots) .
$$

By Lemma 3.3, we have

$$
\xi+n \leq r(y x)=r_{p}(y x) \in L_{+}^{0}(n=1,2, \ldots),
$$

which is again a contradiction. 
Remark 3.1. Let $(S,\|\cdot\|)$ be a complete $R N$ module over $C$ with base $(\Omega, \mathcal{F}, P)$. Then the linear space of all a.s. bounded random linear operators on $S$, denoted by $B(S)$, is an $R N$ module over $C$ with base $(\Omega, \mathcal{F}, P)$ [4]. Define the ring multiplication $: B(S) \times B(S) \rightarrow B(S)$ by $\left(T_{1} \cdot T_{2}\right)(x)=T_{1}\left(T_{2}(x)\right), \forall x \in S$ and $T_{1}, T_{2} \in B(S)$. Then $B(S)$ is a unital complete $R N$ algebra with the identity operator $I$ as the identity.

As an application of the Wintner theorem in unital complete random normed algebras, we have the following Theorem 3.2.

Theorem 3.2. Let $(S,\|\cdot\|)$ be a complete $R N$ module over $C$ with base $(\Omega, \mathcal{F}, P)$. Then there exist no $T_{1}$ and $T_{2}$ in $\mathcal{B}(S)$ such that $T_{1} T_{2}-T_{2} T_{1}=I$.

Remark 3.2. We can see that if the base space $(\Omega, \mathcal{F}, P)$ of the $R N$ module is a trivial probability space, i.e., $\mathcal{F}=\{\Omega, \emptyset\}$, then Theorem 3.1 and Theorem 3.2 automatically degenerate to the corresponding classical cases.

\section{References}

[1] N. Dunford and J. T. Schwartz, Linear Operators, Part I, Interscience, New York, 1957.

[2] T. X. Guo, Extension theorems of continuous random linear operators on random domains, J. Math. Anal. Appl. 193 (1995), no. 1, 15-27.

[3] - The Radon-Nikodým property of conjugate spaces and the w*-equivalence theorem for w*-measurable functions, Sci. China Ser. A 39 (1996), 1034-1041.

[4] Module homomorphisms on random normed modules, Northeast. Math. J. 12 (1996), no. 1, 102-114.

[5] _ A characterization for a complete random normed module to be random reflexive, J. Xiamen Univ. Natur. Sci. 36 (1997), 499-502.

[6] Some basic theories of random normed linear spaces and random inner product spaces, Acta Anal. Funct. Appl. 1 (1999), no. 2, 160-184.

[7] - Representation theorems of the dual of Lebesgue-Bochner function spaces, Sci. China Ser. A 43 (2000), no. 3, 234-243.

[8] Several applications of the theory of random conjugate spaces to measurability problems, Sci. China Ser. A 50 (2007), no. 5, 737-747.

[9] _ Relations between some basic results derived from two kinds of topologies for a random locally convex module, J. Funct. Anal. 258 (2010), no. 9, 3024-3047.

[10] Recent progress in random metric theory and its applications to conditional risk measures, Sci. China Ser. A 54 (2011), no. 4, 633-660.

[11] T. X. Guo and S. B. Li, The James theorem in complete random normed modules, J. Math. Anal. Appl. 308 (2005), no. 1, 257-265.

[12] T. X. Guo and Z. Y. You, A Riesz representation theorem for random inner product modules and its applications, Chinese Ann. Math. Ser. A 17 (1996), no. 3, 361-364.

[13] _ A note on pointwise best approximation, J. Approx. Theory 93 (1998), no. 2, 344-347.

[14] C. E. Rickart, General Theory of Banach Algebras, D. Van Nostrand Company, Inc., 1960.

[15] Y. H. Tang, A new version of the Gleason-Kahane-Zielazko theorem in complete random normed algebras, J. Inequal. Appl. 2012 (2012), 6 pp.

[16] _ Random spectral theorems of self-adjoint random linear operators on complete complex random inner product modules, Linear Multilinear Algebra 61 (2013), no. 3, 409-416.

[17] Y. H. Tang and T. X. Guo, Complete random normed algebras, in press. 
[18] G. Weiss, $B(H)$-commutators: A historical survey, operator theory, Advances and Applications 153 (2004), 307-320

[19] A. Wintner, The unboundedness of quantum-mechanical matrices, Phys. Rev. 71 (1947), $738-739$.

College of Mathematics Physics and Information Engineering

JIAXING UNIVERSITY

JiAXING 314001, P. R. China

E-mail address: tangjohn@126.com 